\title{
La thérapie génique : la recherche progresse !
}

\author{
Gene therapy: research is progressing!
}

\author{
Nassima MOUSSAOUI' \\ ${ }^{1}$ Laboratoire de Pharmacie Galénique. Faculté de Médecine d'Oran. Université d'Oran1
}

\begin{abstract}
Article: Gene therapy: Myth or reality?
Alain Fischer

Paris Descartes-Sorbonne Paris Cite University, Imagine Institute, 75015 Paris, France Comptes Rendus Biologies, 339 (2016) 314-318. DOI: 10.1016/j.crvi.2016.04.011
\end{abstract}

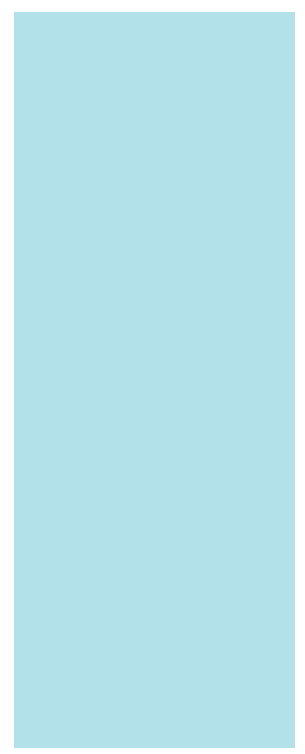

\section{Introduction}

Grâce aux progrès réalisés dans le domaine de la biologie cellulaire et moléculaire, les scientifiques ont développé un ensemble de techniques, qui ont permis de manipuler et de réorganiser les gènes de divers organismes vivants, ce qui a abouti à la mise au point de tout un éventail de nouveaux médicaments.

La biotechnologie moderne s'inscrit dans cette stratégie et regroupe le segment des produits issus de l'ADN recombinant (insuline humaine, somatotropine...) et celui des acides nucléiques qui concerne entre autres la thérapie génique. Le nombre d'essais de thérapie génique approuvés dans le monde a progressivement augmenté ces dernières années, atteignant 116 protocoles par an en 1999 et un total de 2210 protocoles approuvés en 2015[1].

Cet article retrace l'intérêt de la thérapie génique et son bénéfice clinique obtenu au cours des dix-sept dernières années.

Au sens premier, la thérapie génique correspond au remplacement d'un gène défectueux ou absent. Cela revient à remplacer une séquence d'ADN par un gène normal et fonctionnel responsable de la biosynthèse d'une protéine déficiente [2].

Cette technique consiste à introduire dans une cellule, un gène par l'intermédiaire d'un vecteur, dans le génome duquel il est généralement inséré. L'expression de ce gène permet- tra de modifier les propriétés fonctionnelles de la cellule qui devient « transduite ». Cette expression aboutira soit à la restauration d'une fonction perdue ou altérée, soit à l'acquisition d'une nouvelle fonction.

\section{Quelles stratégies et quels vecteurs ?}

Etant donné la faible stabilité des acides nucléiques dans les milieux biologiques et leur faible pénétration intracellulaire,

E-mail : nassima_moussaoui2000@yahoo.fr 
le transfert du matériel génétique dans les cellules peut être réalisé au moyen de virus génétiquement modifiés [3]. En effet, les vecteurs viraux, notamment les adénovirus, les virus adéno-associés (AAV8 et AAV9), les rétrovirus (rétrovirus gam$\mathrm{ma}$ ), ainsi que les lentivirus (en particulier, ceux dérivés du $\mathrm{VIH}$ ) constituent actuellement d'excellents outils biologiques pour le transfert de gènes thérapeutiques d'intérêt.

Par ailleurs, des vecteurs non viraux représentés par les liposomes et les nanoparticules de diamètre inférieur à $1 \mu \mathrm{m}$, font également l'objet de nombreuses recherches pour permettre aux acides nucléiques de rejoindre leur cible [2].

\section{Les applications possibles}

Etant fondée sur l'insertion, la modification, l'inactivation d'un gène ou la correction d'une mutation génétique, il est raisonnable de penser que la thérapie génique est destinée à traiter les maladies héréditaires (hémophilie, dystrophie musculaire, maladie de Gaucher...) mais aussi des maladies acquises : les cancers, la maladie d'Alzheimer et la maladie de Parkinson... [2]

Le premier succès de la thérapie génique a été réalisé autour de l'an 2000 dans le traitement des déficits immunitaires combinés sévères (DICS), caractérisés par un déficit en lymphocytes T. De plus, deux maladies : le déficit immunitaire combiné sévère $X 1$ (SCID X1) et le déficit en adénosine désaminase (ADA), ont été traités avec succès. Cette technologie est actuellement utilisée pour le traitement du Syndrome Wiskott-Aldrich, de l'anémie de Fanconi, des leucodystrophies, de la bêta-thalassémie et de la drépanocytose. Lorsqu'elle a été adaptée à l'œil, la thérapie génique appliquée à un stade précoce, a permis d'améliorer relativement la vision de patients atteints de dystrophie rétinienne héréditaire. Cette même technologie médicale commence à être appliquée avec succès ces cinq dernières années pour le traitement des leucémies à cellules $B$ et des lymphomes.

\section{Conclusion}

Au final, la thérapie génique en tant que technologie innovante, apparait aujourd'hui comme un axe de recherche prometteur qui pourrait ouvrir d'immenses perspectives pour la prise en charge des maladies sans réponse thérapeutique satisfaisante.

\section{Références bibliographiques}

[1] Kenzaburo Tani.Current status ofex vivo gene therapy for hematological disorders: a review of clinical trials in Japan around the world. International Journal of Hematology, (2016) 104: 42-72.

[2] Pascal Wehrlé. Systèmes non viraux pour l'administration des acides nucléiques. Pharmacie galénique : formulation et technologie pharmaceutique 2ème édition (2012) : 272-276.

[3] Hanen Khabou, Deniz Dalkara. La conception de vecteurs adaptés à la thérapie génique oculaire. Medecine/Sciences, (2015) 31: 529-537. 